PREPARED FOR THE U.S. DEPARTMENT OF ENERGY, UNDER CONTRACT DE-AC02-76CH03073

PPPL-3613

PPPL-3613

UC-70

Alpha-particle Measurements Needed

for Burning Plasma Experiments

by

Kenneth M. Young

September 2001

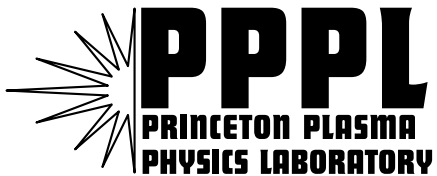

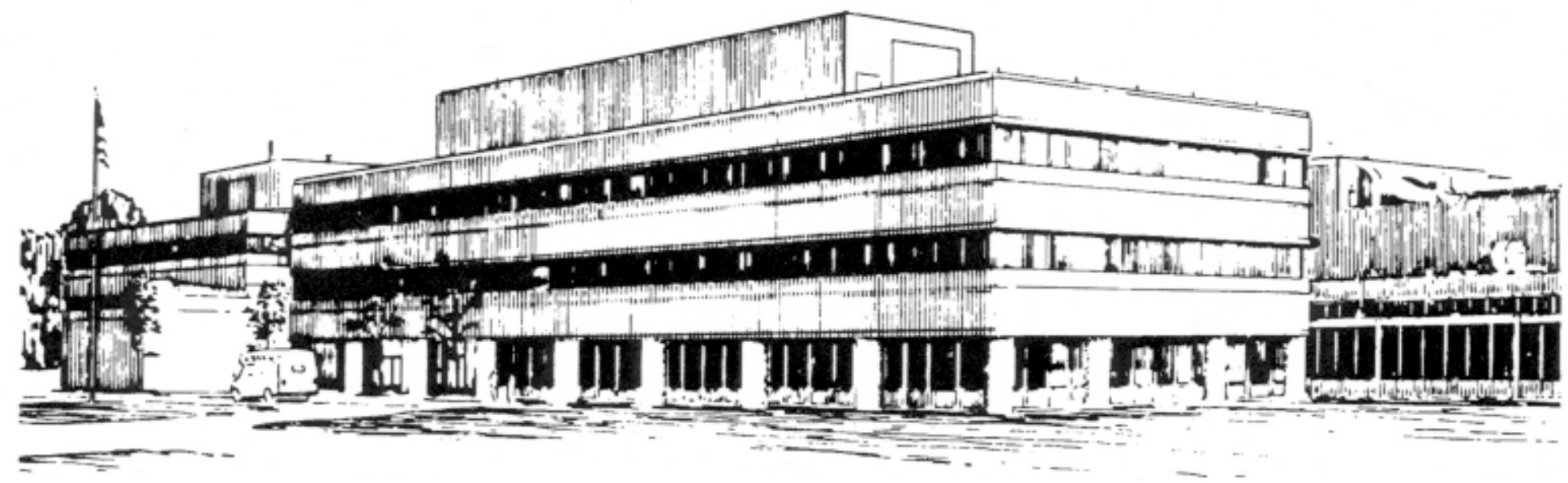

PRINCETON PLASMA PHYSICS LABORATORY PRINCETON UNIVERSITY, PRINCETON, NEW JERSEY 


\section{PPPL Reports Disclaimer}

This report was prepared as an account of work sponsored by an agency of the United States Government. Neither the United States Government nor any agency thereof, nor any of their employees, makes any warranty, express or implied, or assumes any legal liability or responsibility for the accuracy, completeness, or usefulness of any information, apparatus, product, or process disclosed, or represents that its use would not infringe privately owned rights. Reference herein to any specific commercial product, process, or service by trade name, trademark, manufacturer, or otherwise, does not necessarily constitute or imply its endorsement, recommendation, or favoring by the United States Government or any agency thereof. The views and opinions of authors expressed herein do not necessarily state or reflect those of the United States Government or any agency thereof.

\section{Availability}

This report is posted on the U.S. Department of Energy's Princeton Plasma Physics Laboratory Publications and Reports web site in Calendar Year 2001. The home page for PPPL Reports and Publications is: http://www.pppl.gov/pub_report/

DOE and DOE Contractors can obtain copies of this report from:

U.S. Department of Energy

Office of Scientific and Technical Information

DOE Technical Information Services (DTIS)

P.O. Box 62

Oak Ridge, TN 37831

Telephone: (865) 576-8401

Fax: (865) 576-5728

Email: reports@adonis.osti.gov

This report is available to the general public from:

National Technical Information Service

U.S. Department of Commerce

5285 Port Royal Road

Springfield, VA 22161

Telephone: 1-800-553-6847 or

(703) 605-6000

Fax: (703) 321-8547

Internet: http://www.ntis.gov/ordering.htm 


\title{
ALPHA-PARTICLE MEASUREMENTS NEEDED FOR BURNING PLASMA EXPERIMENTS
}

\author{
Kenneth M. Young*
}

\section{INTRODUCTION}

The next major step in magnetic fusion studies will be the construction of a burning plasma (BP) experiment where the goals will be to achieve and understand the plasma behavior with the internal heating provided by the fusion-generated alphaparticles ( $\alpha$-particles). Two devices with these physics goals have been proposed, the International Thermonuclear Experimental Reactor (ITER) ${ }^{1}$ and the Fusion Ignition Research Experiment (FIRE) $)^{2}$. Extensive conceptual design work for the instrumentation to try to meet the physics demands has been done for these devices, especially ITER ${ }^{3}$, and the overall requirements are reviewed in this book ${ }^{4}$.

This article provides a new look at the measurements specifically important for understanding the physics aspects of the $\alpha$-particles. An earlier article addressed a similar topic ${ }^{5}$, but two significant events have occurred since then. The first was the completion of physics experiments on $\mathrm{JET}^{6}$ and $\mathrm{TFTR}^{7}$ with deuterium-tritium (DT) fueling, and the first chances to study $\alpha$-physics ${ }^{8,19}$, and the second is the realization that relatively compact plasmas, making use of advanced tokamak plasma concepts, are the most probable route to burning plasmas and ultimately a fusion reactor. These plasmas require measurements with excellent spatial and temporal resolutions and a large number of diagnostic signals will feed into the control of the plasmas.

The BP experiment will provide the science basis for the operation of a fusion reactor. Thus it is imperative that the understanding developed should lead to optimization of the plasma performance and being able to use the $\alpha$-particles in controlling the plasma. The $\alpha$-particles will become the dominant heating term in any successful BP experiment, and as such will play a key role in the physics of the plasma. Their generation in the core can be expected to significantly affect the density and

\footnotetext{
* Kenneth M. Young, Princeton Plasma Physics Laboratory, Princeton, NJ 08543,
} U.S.A. 
pressure profiles associated with the confinement regime of choice. The fusion output in the form of these $\alpha$-particles has got to be fed back into the control of the heating to affect the pressure gradients; it must also be used to assist the current profile, and possible provide current drive; and it must provide momentum to affect the shear flow with its strong influence on the transport properties. These roles then set the purpose for the $\alpha$-particle measurements, setting up the requirements for specific diagnostics. Table 1 provides a listing of the diagnostic techniques which are being considered for the $\alpha$-physics measurements in a BP device. They are discussed in the following sections, but some comments indicate where testing on operating devices is very desirable. Many of the diagnostics necessary for these measurements are not fully developed or will be severely constrained in their operation under BP conditions.

\section{MEASUREMENTS OF CONFINEMENT AND SLOWING DOWN: EFFECT OF PLASMA HEATING AND PROFILES}

The most important measurements to be made for understanding the heating impact of the $\alpha$-particles are the profile measurements of the electron temperature and ion temperature. The pioneering measurements on $\mathrm{TFTR}^{8}$ and $\mathrm{JET}^{19}$ were relatively difficult because of the low-Q and thus small temperature increments. In TFTR averages over a number of shots were required to establish profile differences. The BP plasmas are expected to be created with reversed shear, for which there is little data from TFTR or JET in DT plasmas, so very good measurements of these key parameters, and the safety factor, $\mathrm{q}(\mathrm{r})$, will be required.

The best measurement of the source $\alpha$-particles generated in the fusion reactions will be the $14 \mathrm{MeV}$ neutron flux, with collimation provided to obtain spatial dependence. But it will be necessary to follow the history of the confined $\alpha$-particles as they lose energy and are distributed across the plasma radius. It is unfortunately not possible to rely on a single measurement technique for this history because of limitations on each of the techniques so far proposed. Collective Thomson scattering may be the best technique, but, so far, has only been successfully demonstrated on the more easily measured thermal plasma ${ }^{10}$. Measurement in the microwave range gives the possibility of relatively good spatial resolution because the scattering angle can be quite large, and the hardware components are mostly available. Unfortunately, there are very limited transmission windows between resonant harmonics across the plasma, which constrains the measurement and causes high background noise levels. At high densities refraction will be an issue. The other possible choice for which sources and detectors are available is a $\mathrm{CO}_{2}$ laser at $10 \mu$, and a test is planned for studying the fast ions generated by the JT-60U negative-ion-based neutral beam ${ }^{11}$. In this case, though, the scattering angle must be very small $\left(<1_{i}\right)$ making spatial resolution and accessibility to the plasma very problematic. A compromise solution would be in the far infra-red (FIR) spectral region, using technologically feasible lasers, but for which developments in the lasers and detectors are necessary.

Neutral particle analyzers have proven capable of obtaining core $\alpha$-particle 
Table 1 Summary of diagnostics for alpha-particle physics

\begin{tabular}{l}
$\begin{array}{l}\text { Diagnostics for alpha-particle } \\
\text { physics }\end{array}$ \\
\hline Confinement and Slowing Down* \\
\hline $14 \mathrm{MeV}$ Neutron profile for $\alpha$-source \\
Collective scattering \\
$\alpha$-CHERS \\
High-energy neutral particle analyzers \\
Knock-on neutron measurement \\
(neutron spectrometer or bubble chamber) \\
Impact on Plasma Stability* \\
Mirnov coils (high frequency) \\
Correlation reflectometry \\
Alfv n antennas \\
Beam emission spectroscopy \\
Ion cyclotron emission \\
Instabilities affecting redistribution and \\
losintillator, diamond)
\end{tabular}

Comments relevant to a

burning plasma (BP) device

Proven technique; needs same spatial

resolution as $\mathrm{T}_{\mathrm{i}}(\mathrm{r})$.

Demonstration required (JET, JT-60U); spatial, energy res. concerns.

Proven technique; needs neutral beam; radiation-effects concerns.

Requires low-Z impurity (JET); spatial resolution is an issue.

Difficult to get temporal/spatial resolution (JET).

Proven technique, but tough radiation environment.

Rapidly evolving technique (JET)

Valuable for JET physics studies. Necessary

for BP?

Proven technique; needs neutral beam.

Proven measurement; has it any value?

Proven technique.

Proven technique.

For energy, source measurement, need scintillator but neutron, thermal issues (JET); diamond demonstration needed (JET).

Proven technique; needs neutral beam.

Proven technique.

2.5 MeV neutrons to be measured in larger $14 \mathrm{MeV}$ neutrons (JET).

Proven technique; needs neutral beam. Prototyping needed (DIII-D, JET)

\footnotetext{
* Assumes that there are good measurements of $n_{e}(r), T_{e}(r), T_{i}(r), v(r), q(r)$, magnetic equilibria, etc.
} 
distribution functions in two somewhat different applications. In JET, Gondhalekar et al. made use of the enhanced charge-exchange cross-sections of natural low-Z impurities, $\mathrm{Be}$ and $\mathrm{C}$, in the core in unfolding the spectrum of $\alpha$-particles from the combined $\mathrm{D}$ - and $\alpha$-spectra ${ }^{9}$. There was only one vertical line of sight providing a lineintegral measurement, and it is to be hoped that a future JET measurement will have additional sightlines. On TFTR, the novel pellet charge exchange (PCX) concept, using a neutral particle analyzer in conjunction with a low-Z lithium pellet radially-injected into the plasma with horizontal observation was applied ${ }^{13}$. The pellet flight time provided the spatial resolution for the $\alpha$-particle distribution function and showed redistribution of trapped $\alpha$-particles in reversed-shear discharges. In high-density plasmas with neutral beam heating, the failure of the pellet to penetrate limits its applicability. A spectroscopic technique making use of the charge exchange of the $\alpha$ particles with neutral beam particles, the so-called $\alpha$-CHERS (charge exchange recombination spectroscopy), was also used on $\mathrm{TFTR}^{8}$. The high-energy tail of the $468.6 \mathrm{~nm} \mathrm{He}^{+}$line is spectrally resolved to determine the slowing-down spectrum of the $\alpha$-particles below about four-times the neutral beam energy (i.e. up to about $700 \mathrm{keV}$ in the TFTR case). One result was that the anomalous diffusivity of the $\alpha$-particles was less than $0.03 \mathrm{~m}^{2} / \mathrm{s}$. This measurement of the tail requires very precise knowledge of the visible bremsstrahlung background and other noise sources. The principal of operation of this technique is the same as that used for measurement of the He-ash or the ion temperature and plasma rotation, though the instrument requires a higher optical throughput. All these instruments depend on a neutral beam source, with energy in the range $100-150 \mathrm{keV} / \mathrm{amu}$. Particles with this beam energy do not penetrate well into the proposed burning plasmas and development of a short-pulse intense diagnostic neutral beam has been proposed ${ }^{13}$.

The high-energy tail of the neutron spectrum contains a "knock-on" component created by the $\alpha$-particles colliding with the fusing atoms and imparting additional center-of-mass velocity, leading to higher energy neutrons. The spectrum of this very small tail, tied to the $\alpha$-energy spsectrum, approximately $10^{-3}$ of the peak, has been measured on JET by a neutron spectrometer, averaging over many DT shots ${ }^{14}$. It is difficult to see how such a spectrometer could provide sufficient counts for both adequate temporal or spatial resolution. Hence a development of a set of pulsed bubble chamber detectors, with very sharp low-energy cut-offs, the lowest at about $15 \mathrm{MeV}$, so that only the "knock-on" tail's spectrum is measured, has been proposed ${ }^{15}$. The detectors could be installed in collimator tubes of a neutron camera for a BP device.

Measurement of escaping $\alpha$-particles, while important for the issue of $\alpha$-particle confinement is described in section 4 .

\section{IMPACT ON PLASMA STABILITY; MACROSTABILITY AND COLLECTIVE EFFECTS}

Fast ions and $\alpha$-particles have very significant impacts on the plasma in that they can stabilize MHD modes and destabilize a large variety of high-frequency $(>100 \mathrm{kHz})$ 
modes of the Alfv $n$ eigenmode (AE) type. The behavior of low-frequency MHD modes can be relatively easily followed by the Mirnov coils. The normal pressure diagnostics must be monitored closely to observe any build-up of the pressure.

High-frequency Mirnov coils give a clear picture of the presence of the various AE instabilities, being particularly good at following the evolution in frequency (chirping, etc.) which have provided the necessary clues for mode identification. In BP reversedshear plasmas a build-up of an avalanche of these instabilities to cover a large fraction of the plasma cross-section, providing a clear rapid transport path, is a concern. Correlation reflectometry on TFTR has been effective in spatially localizing modes and providing a resource for theoretical analysis and prediction ${ }^{16}$. Since its sensitivity to small amplitude density fluctuations is much greater, it can detect fluctuations not visible on the Mirnov coils. For a BP plasma, it is essential that there be coverage across the full plasma cross-section, so that a combination of $\mathrm{O}$ - and $\mathrm{X}$-mode correlation reflectometry is required.

Another opportunity for studying AE modes has been taken at JET where a set of external coils has been used to destabilize stable AE modes, hence allowing for study of the growth of the modes and tying behavior closely to theoretical projection ${ }^{17}$. It is not clear that there is value to extending this type of experiment to a BP where the internal drives are likely to be very strong.

Another very clear instability observed on TFTR and JET occurs at harmonics of the ion cyclotron frequency at the outside, low- $\mathrm{B}_{\mathrm{T}}$, plasma edge, caused by trapped $\alpha$ particles interacting with the low plasma density ${ }^{8,9,18}$. The interactions are so complex that it is doubtful whether any meaningful information about the $\alpha$-particle population will be obtained in a BP at high magnetic field.

\section{IMPACT OF INSTABILITIES ON THE ALPHA-PARTICLE BEHAVIOR; REDISTRIBUTION AND LOSS}

Good measurements of the MHD and the higher frequency MHD modes are required and internal $T_{e}$ fluctuation measurements are particularly important. In TFTR DT experiments, the possibility of investigating the redistribution of the $\alpha$-particles was provided by the two diagnostics, PCX and $\alpha$-CHERS ${ }^{8}$. Sawteeth were shown to redistribute the trapped and non-thermal $\alpha$-particles and TAE modes redistributed trapped $\alpha$-particles. The key to the measurements was the good spatial resolution of the instruments.

The limit to redistribution is loss, which must be measured effectively with an array of detectors. Surface temperature measurements by infra-red imaging gives good spatial information about the overall energy loss of fast ions. But localized detectors are necessary for obtaining information about the energy and source of the $\alpha$-particles. An array of such detectors in the lower-outside quadrant (depending on the toroidal field direction) of the first wall can provide this information. On TFTR, an array of thin-scintillator detectors behind shielded apertures gave very specific data on the pitch angle and gyroradius of the lost particles ${ }^{8}$. In quiescent plasmas, the losses could be 
assigned to first orbit losses and toroidal field ripple induced loss. In discharges with active MHD, and with the total light being fed to a photomultiplier, quantitative measurements of the losses were obtained for sawteeth, fishbones, locked modes, tearing modes, the thermal quench prior to major plasma current disruptions, highfrequency kinetic ballooning modes and ICRF heating. For application in a BP device, improved scintillators which can tolerate high temperatures and radiation-hard optics must be developed. An alternative technique using Faraday cup detectors has been proposed and installed on $\mathrm{JET}^{19}$. These detectors are easier to locate and install, requiring only electrical wiring, but they only provide information about the energy of the $\alpha$-particles passing through the aperture.

\section{MEASUREMENT FOR STUDIES OF TRANSPORT OF FAST IONS AND $\alpha$ - ASH REMOVAL}

The measurement of helium in the core of the plasma has been carried out spectroscopically in many tokamaks to study the diffusion of this impurity ${ }^{7}$. In a BP this $\alpha$-ash builds up as the $\alpha$-particles thermalize and for a reactor could stop the burning process. Hence it is critical to measure it both in the plasma core and in the efflux through the divertor. In the core, charge-exchange spectroscopy, using the full $468.6 \mathrm{~nm} \mathrm{He}{ }^{+}$spectral line, is the only available technique. Again, there is a requirement for a neutral beam. In the divertor, residual gas analysis and Penning gauges can be used for quantifying the helium flow from the plasma.

One expects that there will be specific experiments aimed at manipulating the $\alpha$ particles, to be able to enhance the $\alpha$-ash loss ${ }^{7}$ or to channel the high-energy particles ${ }^{7}$ toward the goals of controlling plasma behavior. Suggestions for affecting the current drive and the plasma rotation and shear have been made and have to be studied much more deeply than in preliminary TFTR and JET experiments ${ }^{7,6}$. The diagnostics required for these physics studies will be the standard set of profile diagnostics, and magnetic measurements for studies of current drive.

An associated aspect of controlling the $\alpha$-particle behavior is the fueling of the hydrogen isotopes, deuterium and tritium. The measurement of the core densities of these ions can be done spectroscopically, with a neutral beam to provide the source of particles for the neutralization. There are proposals that the relative densities could be obtained by neutron spectroscopy, even though the DD neutron intensity will be many orders of magnitude smaller than the DT intensity, and it is hoped to test this idea on JET. An alternative technique which uses simple coils to carry out fast-wave reflectometry was prepared for test on DIII-D and is awaiting test ${ }^{13}$.

\section{SUMMARY AND RECOMMENDATIONS FOR MEAUREMENTS FOR $\alpha$ - PHYSICS IN A BP DEVICE}

We have briefly described four areas of physics study to be carried out in burning plasma experiments for understanding the behavior of the heating particles, 
emphasizing the measurements needed and examples of diagnostics for carrying out those measurements. The set of diagnostics must be aimed at achieving the mission of the BP device, the ultimate goal of which must be establishing the optimal operating conditions for the more-engineering-oriented next step. For the latter, access to the plasma for diagnostics will necessarily be more restricted and plasma control, rather than physics understanding, will be the prime motivator for the instrumentation.

The set of diagnostics to enable thorough $\alpha$-physics studies that can be considered now for implementation on a BP device is shown in table 1. It is essential that there be very high quality diagnostics for measuring the profiles of the main plasma parameters and for measuring the fluctuations over a wide range of frequencies. Many of the techniques with the best spatial resolution are dependent on a neutral beam, which is a major challenge for high-density and large tokamak plasmas. Good spatial and temporal resolution neutron measurements will be key, particularly for confinement studies, since they will define the $\alpha$-source. Good measurements of the fluctuations, externally with many Mirnov coils and internally with many spatial locations of ECE and reflectometry to provide local temperature and density variations are essential for defining the instability modes.

Finally a set of diagnostics for the $\alpha$-particles themselves must be installed on a BP device. Physics studies of confinement and redistribution demand techniques with very good spatial resolution, such as was available with $\alpha$-CHERS and PCX on TFTR. Both of these diagnostics are difficult to implement on a BP device, the former needing a neutral beam and the latter a high-speed lithium pellet. Collective Thomson scattering techniques and neutral particle analysis dependent on natural levels of low- $\mathrm{Z}$ impurities must be developed further, but it is difficult to see how they can provide sufficiently good spatial resolution to resolve some of the physics issues. An array of lost- $\alpha$ detectors at the wall to give energy and source information about the escaped particles will enhance the physics understanding. All these techniques should, if possible, be applied in a second JET DT campaign to determine the value of installing them on a BP device.

\section{ACKNOWLEDGEMENTS}

I am very grateful for many discussions with and guidance from Raffi Nazikian. This work was supported by U.S. DOE Contract No. DE-AC02-76CH0307.

\section{REFERENCES}

1. R. Aymar, The ITER Joint Central Team, ITER-FEAT - The future international burning plasma experiment overview", in Proceedings of $18^{\text {th }}$ IAEA Fusion Energy Conference, Sorrento (IAEA, Vienna, 2001) paper IAEA-CN-77/OV/1, http://www.iaea.org/programmes/ripc/physics/fec2000/html/fec2000.htm.

2. D.M. Meade. S.C. Jardin, C.E. Kessel, M.A. Ulrickson, J.H. Schultz, P.H. Rutherford, et al., Mission and design of the Fusion Ignition Research Experiment (FIRE), in Proceedings of $18^{\text {th }}$ 
IAEA Fusion Energy Conference, Sorrento (IAEA, Vienna, 2001) paper IAEA-CN-77/FTP2/16, http://www.iaea.org/programmes/ripc/physics/fec2000/html/fec2000.htm.

3. ITER Physics Expert Group on Diagnostics, ITER Physics Basis Editors, ITER physics basis, Chapter 7: Measurement of plasma parameters, Nucl. Fusion 39 (12), 2541 - 2575 (1999).

4. A.E. Costley, in: Advanced Diagnostics for Magnetic and Inertial Fusion, edited by P.E. Stott and G. Gorini (Plenum Press, New York, 2001) this volume.

5. S.J. Zweben, S. Putvinski, M.P. Petrov, G. Sadler, K. Tobita and K.M. Young, in: Diagnostics for Experimental Thermomnuclear Fusion Reactors, edited by P.E. Stott, G. Gorini and E. Sindoni (Plenum Press, New York, 1996) pp 467 - 476.

6. M. Keilhacker, A. Gibson, C. Gormezano, P.J. Lomas, P.R. Thomas, M.L. Watkins et al., High fusion performance from deuterium plasmas in JET, Nucl. Fusion, 39 (2), $209-234$ (1999).

7. R.J. Hawryluk, Results from deuterium-tritium tokamak confinement experiments, Rev. Mod. Phys., 70 (2), $537-587$ (1998).

8. S.J. Zweben, R.V. Budny, D.S. Darrow, S.S. Medley, R. Nazikian, B.C. Stratton et al., Nucl. Fusion 40 (1), $91-149$ (2000).

9. JET Team (prepared by P.R. Thomas), Alpha particle studies during JET DT experiments, Nucl. Fusion, 39 (11Y), 1619 - 1625 (1999).

10. H. Bindslev in: Advanced Diagnostics for Magnetic and Inertial Fusion, edited by P.E. Stott and G. Gorini (Plenum Press, New York, 2001) this volume.

11. T. Kondoh, S. Lee, D.P. Hutchinson and R.K. Richards, Collective Thomson scattering using a pulsed $\mathrm{CO}_{2}$ laser in JT-60U, Rev. Sci. Instrum., 72 (1), 1143 - 1146 (2001).

12. R.K. Fisher, H.H. Duong, J.M. McChesney, P.B. Parks, S.S. Medley, R.V. Budny et al., Alpha diagnostics using pellet charge exchange: results in the Tokamak Fusion Test Reactor and prospects for ITER, Rev. Sci. Instrum., 68 (1), 336 - 339.

13. A.E. Costley, D.J. Campbell, S. Kasai, K.M. Young amd V. Zaveriaev, ITER R\&D: Auxiliary Systems: Plasma Diagnostics, Fus. Engg. \& Des., 55 (2-3), 331 - 346 (2001).

14. J. K llne, L. Ballabio, J. Frenje, S. Conroy, G. Ericsson, M. Tardocchi, E. Traneus and G. Gorini, Observation of the alpha particle "knock-on" neutron emission in magnetically confined DT fusion plasmas, Phys. Rev. Lett., 85 (6) 1246 - 1249 (2000)

15. R.K. Fisher, P.B. Parks, J. Liptac, D.C. Ingram, C.E. Brient, S.S. Medley et al., Measured response of bubble neutron detectors and prospects for alpha knock-on diagnostics, Rev. Sci. Instrum., 72 (1), 796 - 800 (2000).

16. R. Nazikian and E. Mazzucato, Reflectometer measurements of density fluctuations in tokamak plasmas, Rev. Sci. Instrum., 66 (1), 392 - 398 (1995).

17. D. Testa and A. Fasoli, The effect of plasma shaping on the damping of low $n$ Alfv $n$ eigenmodes in JET tokamak plasmas, Nucl. Fusion, 41 (7), $809-812$ (2001).

18. N.N. Gorelenkov and C.Z. Cheng, Alfv n cyclotron instability and ion cyclotron emiss ion, Nucl. Fusion, 35 (12), 1743 - 1752 (1995).

19. F.E. Cecil, B. Roy, C. Sutton and N. Wasinger, A thin film device as a low energy, high flux charged particle spectrometer, Rev. Sci Instrum., 72 (1), 792 - 795 (2001). 


\section{External Distribution}

Plasma Research Laboratory, Australian National University, Australia

Professor I.R. J ones, Flinders University, Australia

Professor J oão Canalle, Instituto de Fisica DEQ/IF - UERJ , Brazil

Mr. Gerson O. Ludwig, Instituto Nacional de Pesquisas, Brazil

Dr. P.H. Sakanaka, Instituto Fisica, Brazil

The Librarian, Culham Laboratory, England

Library, R61, Rutherford Appleton Laboratory, England

Mrs. S.A. Hutchinson, JET Library, England

Professor M.N. Bussac, Ecole Polytechnique, France

Librarian, Max-Planck-Institut für Plasmaphysik, Germany

J olan Moldvai, Reports Library, MTA KFKI-ATKI, Hungary

Dr. P. Kaw, Institute for Plasma Research, India

Ms. P.J . Pathak, Librarian, Insitute for Plasma Research, India

Ms. Clelia De Palo, Associazione EURATOM-ENEA, I taly

Dr. G. Grosso, Instituto di Fisica del Plasma, Italy

Librarian, Naka Fusion Research Establishment, J AERI, J apan

Library, Plasma Physics Laboratory, Kyoto University, J apan

Research Information Center, National Institute for Fusion Science, J apan

Dr. O. Mitarai, Kyushu Tokai University, J apan

Library, Academia Sinica, Institute of Plasma Physics, People's Republic of China

Shih-Tung Tsai, Institute of Physics, Chinese Academy of Sciences, People's Republic of China

Dr. S. Mirnov, TRINITI, Troitsk, Russian Federation, Russia

Dr. V.S. Strelkov, Kurchatov Institute, Russian Federation, Russia

Professor Peter Lukac, Katedra Fyziky Plazmy MFF UK, Mlynska dolina F-2, Komenskeho Univerzita, SK-842 15 Bratislava, Slovakia

Dr. G.S. Lee, Korea Basic Science Institute, South Korea

Mr. Dennis Bruggink, Fusion Library, University of Wisconsin, USA

Institute for Plasma Research, University of Maryland, USA

Librarian, Fusion Energy Division, Oak Ridge National Laboratory, USA

Librarian, Institute of Fusion Studies, University of Texas, USA

Librarian, Magnetic Fusion Program, Lawrence Livermore National Laboratory, USA

Library, General Atomics, USA

Plasma Physics Group, Fusion Energy Research Program, University of California at San Diego, USA

Plasma Physics Library, Columbia University, USA

Alkesh Punjabi, Center for Fusion Research and Training, Hampton University, USA

Dr. W.M. Stacey, Fusion Research Center, Georgia Institute of Technology, USA

Dr. J ohn Willis, U.S. Department of Energy, Office of Fusion Energy Sciences, USA

Mr. Paul H. Wright, Indianapolis, Indiana, USA 
The Princeton Plasma Physics Laboratory is operated by Princeton University under contract with the U.S. Department of Energy.

\author{
Information Services \\ Princeton Plasma Physics Laboratory \\ P.O. Box 451 \\ Princeton, NJ 08543
}

Phone: 609-243-2750

Fax: 609-243-2751

e-mail: pppl_info@pppl.gov

Internet Address: http://www.pppl.gov 